\title{
Aprendizaje-servicio y tecnologías digitales: ¿una relación posible?
}

\section{(Service-learning and digital technologies: a possible relationship?)}

\author{
Anna Escofet \\ Universidad de Barcelona (España)
}

DOI: http://dx.doi.org/10.5944/ried.23.1.24680

\section{Cómo referenciar este artículo:}

Escofet, A. (2020). Aprendizaje-servicio y tecnologías digitales: ¿una relación posible? RIED. Revista Iberoamericana de Educación a Distancia, 23(1), pp. 169-182. doi: http://dx.doi.org/10.5944/ried.23.1.24680

\section{Resumen}

El uso generalizado de las tecnologías digitales junto con la extensión de las redes sociales ha creado nuevos espacios de comunicación y de encuentro en los que personas y actores sociales y políticos entran en interconexión. Ello abre espacios y posibilidades diversas de participación digital de manera más accesible, instantánea, continuada, igualitaria y personalizada. Las tecnologías digitales facilitan, en primer lugar, la distribución y el acceso a la información, lo que permite acceder de manera autónoma y sencilla al ámbito público sin depender de la intermediación de las organizaciones informativas profesionales. En segundo lugar, permiten la comunicación interactiva, lo cual las convierte en un instrumento de interpelación directa que propicia la revitalización de las prácticas deliberativas que forman parte del ideal democrático. En tercer lugar, la interacción propiciada por las tecnologías digitales facilita la organización y eficacia de redes cívicas y espacios de movilización ciudadana. Finalmente, la participación mediada por las tecnologías digitales tiene implicaciones para la identidad personal y social, convirtiendo a las personas en ciudadanos del mundo y en miembros de una comunidad en la que el tiempo y el espacio desaparecen, confrontando actitudes y valores de distintos grupos que están obligados a redefinir sus concepciones para arbitrar su participación. Unir las tecnologías digitales con proyectos de aprendizaje-servicio facilita que jóvenes, niñas y niños experimenten de forma real la solidaridad, la responsabilidad y el servicio a los otros, así como la satisfacción por los objetivos conseguidos. En definitiva, facilita conseguir el desarrollo de una ciudadanía responsable y consciente.

Palabras clave: aprendizaje-servicio; tecnologías digitales; ciudadanía digital. 


\begin{abstract}
The widespread use of digital technologies -together with the extension of social mediahas created new communication and meeting spaces that interconnect people and social or political actors. This opens up different spaces and possibilities in a more accessible, immediate, continued, egalitarian and personalized way. Digital technologies facilitate the access to information and its distribution, a gateway to the public sphere in a more autonomous and simpler way, which does not rely on the intermediation of professional information organizations. They allow interactive communication creating an instrument of direct questioning. Therefore, the revitalization of deliberative practices that are part of the democratic ideal is encouraged. This interaction led by digital technologies facilitates the organization of civic networks together with spaces of citizen mobilization. Such participation has implications for personal and social identity, as it creates citizens of the world and members of a community that is not subjected to time and space. Hence, attitudes and values of different groups are confronted, compelling them to redefine their conceptions in order to arbitrate citizen participation. When combined, digital technologies and service-learning projects allow children and youth to experience authentic solidarity, responsibility and service to others, as well as the satisfaction for the goals achieved. In a nutshell, it facilitates the development of responsible and conscious citizenship.
\end{abstract}

Keywords: service-learning; digital technologies; digital citizenship.

La influencia de las tecnologías digitales en todos los ámbitos de nuestra sociedad es algo que está ya fuera de toda duda. En el caso de niños, niñas y jóvenes (Turkle, 1997; Tapscott, 1998), se han generado nuevas prácticas en las actividades en la red. Estas tendencias, que se interrelacionan y se potencian, tienen que ver con la ubicuidad, las nuevas formas de participación en la comunidad, la creación de nuevos tipos de contenido, y la gestión y el acceso a la información.

En primer lugar, la ubicuidad hace referencia a la invisibilidad de los dispositivos tecnológicos, de manera que los ordenadores, los teléfonos móviles y las tabletas se adaptan a las necesidades y a las formas de vivir de las personas. Los datos muestran que los chicos y las chicas son más propensos a tener un teléfono móvil que a tener un ordenador de sobremesa, y estos teléfonos están proporcionando una variedad de actividad en Internet cada vez más elevada y una frecuencia de conexión más ininterrumpida. Además, la ubicuidad de las tecnologías móviles facilita que las personas jóvenes puedan acceder a cualquier contenido inmediatamente.

En segundo lugar, en cuanto a las nuevas formas de participación en comunidad, Internet ha creado las llamadas comunidades virtuales, que liberan de las limitaciones de la proximidad geográfica y de diversas características sociales. Lo que caracteriza a las comunidades virtuales, además de la superación de las barreras de espacio y tiempo, es un objetivo común por el que se expanden las interacciones en la red y se potencian nuevas formas de comunicación, que a la vez alientan el compromiso de 
desarrollar aún más las relaciones en la red y las propias comunidades (McCorkindale, DiStaso y Sisco, 2013).

En tercer lugar, en cuanto a la creación de nuevos tipos de contenido, las tecnologías digitales ponen a disposición de usuarios y usuarias diferentes herramientas y espacios virtuales que permiten la creación y la difusión de información y de contenidos a nivel global, desde la elaboración de una página web, pasando por la creación y/o la participación en blogs, hasta la compartición de vídeos y fotos o la mezcla de contenidos en línea para hacer otros nuevos. Los contenidos audiovisuales se han unido con los contenidos textuales y han provocado que se puedan reproducir, manipular y redistribuir muy fácilmente. Internet y las tecnologías que permiten la edición digital han facilitado las herramientas para crear, mezclar y compartir contenidos en una escala que anteriormente sólo había sido accesible a especialistas y profesionales de la transmisión y edición de contenidos.

Y, finalmente, está claro que Internet, con motores de búsqueda basados en algoritmos, ha hecho que el acceso a la información sea muy fácil y que se pueda encontrar cualquier información en los buscadores de manera rápida: la gestión y la búsqueda de contenidos e información se hace mediante palabras clave y mediante hipertextos e hipermedia, donde palabras y contenidos audiovisuales están unidas electrónicamente mediante múltiples cadenas o recorridos en una textualidad abierta (Landow, 2009).

De este modo aparece el concepto de sociedad 2.0 (Galindo, 2009) en la cual la comunicación entre individuo, instituciones y comunidad es directa al estar mediada por las tecnologías digitales. Banaji y Buckingham (2013), por su parte, han analizado el impacto que han tenido las redes sociales en la participación ciudadana, revisando los cambios provocados y las nuevas modalidades de participación creadas. Así, por ejemplo, en nuestros días es posible enviar al ayuntamiento de nuestra localidad mediante una aplicación en el teléfono móvil una fotografía de un espacio especialmente sucio o degradado, y también es posible realizar una votación virtual para decidir el nombre de una plaza o de una calle. Estos son ejemplos de cómo las redes sociales pueden facilitar la participación y, con ella, promover la consecución de la ciudadanía activa y el compromiso cívico. Pero ċen qué consiste la participación digital?

\section{Tecnologías digitales y participación digital}

Las tecnologías digitales y las redes sociales crean nuevos espacios de comunicación y encuentro en los que personas y actores sociales y políticos se relacionan y entran en interconexión (Mossberger, Tolbert y McNeal, 2008). Ello abre espacios y posibilidades diversas de participación digital, de manera más accesible, instantánea, continuada, igualitaria y personalizada (Buckingham, 2005).

Es así como las tecnologías permiten establecer vínculos de una persona o un colectivo con otras personas con inquietudes y reivindicaciones similares y trasladar 
a los espacios virtuales el proceso de debate y deliberación llevado a cabo en los entornos físicos. Es por ello por lo que se abren espacios de oportunidades, nuevos hábitos de participación de la ciudadanía, que encuentran en las tecnologías digitales nuevas maneras de dar respuesta a sus necesidades, dando lugar a lo que se han denominado usos cívicos de las tecnologías digitales (Martínez, 2011), que se desglosan a continuación.

Las tecnologías digitales facilitan, en primer lugar, la distribución y el acceso a la información, lo que permite acceder de manera autónoma y sencilla al ámbito público sin depender de la intermediación de las organizaciones informativas profesionales. En segundo lugar, las tecnologías digitales permiten la comunicación de manera interactiva, lo que las convierte en un instrumento de interpelación directa y además puede propiciar la revitalización de las prácticas deliberativas que forman parte del ideal democrático. En tercer lugar, la interacción propiciada por las tecnologías digitales facilita la organización y la eficacia de redes cívicas y espacios de movilización ciudadana. Finalmente, la participación digital tiene implicaciones positivas para la identidad personal y social, al propiciar a las personas la conversión en ciudadanas del mundo -que comparten un mismo espacio de interacción- y en miembros de una comunidad en la que el tiempo y el espacio desaparecen, confrontando actitudes y valores de distintos grupos que están obligados a redefinir sus concepciones para arbitrar su participación de manera horizontal.

Por todo ello podemos afirmar que las tecnologías digitales se presentan como el camino para potenciar la implicación política, la participación en actividades de voluntariado, la solución colectiva de problemas comunitarios y las actividades de protesta política (Reig, 2012). Y ello es posible, por ejemplo, dado que tal y como señalan Álvaro y Rubio (2016), las redes sociales permiten segmentar los problemas, convertirlos en microproblemas y afrontarlos desde microsoluciones, diseñando estrategias, repartiendo tareas, compartiendo resultados mediante la formación de auténticas comunidades cívicas.

En definitiva, las potencialidades de las tecnologías digitales se nos muestran como elevadas, tanto con relación al acceso al conocimiento -que puede realizarse de un modo crítico y emancipador gracias a la facilidad para el acceso a la información y la posibilidad de contrastar la veracidad de aquello que se recibe- como en el fomento de la participación cívica.

Somos conscientes de que todo ello no se produce por generación espontánea y que la información -y las comunicaciones- pueden ser manipuladas y tergiversadas desde núcleos de poder, pero defendemos la necesidad de educar en un uso consciente y crítico de las tecnologías digitales para combatir prácticas manipulativas e interesadas. En este sentido, Gozálvez (2011) hace diversas propuestas pedagógicas dirigidas a la educación formal, alentando un pensamiento crítico y creador desde la cooperación y la interactividad. Considera que las instituciones educativas tienen que abrirse a las tecnologías digitales, tanto como mecanismo de aprendizaje y de 
búsqueda de conocimiento como oportunidad para reflexionar acerca de sus usos sociales. Para ello propone integrar valores como:

- la libertad, analizando espacios digitales para la participación cívica y pacífica; considerando las consecuencias de los propios actos sobre la privacidad y libertad de las demás personas; reflexionando acerca de los peligros para la propia intimidad o integridad que encierran ciertas prácticas en la red; fomentando el pensamiento crítico; estudiando de qué modo el acceso a la información y a la tecnología comunicativa aumenta las capacidades de los pueblos y las personas para vivir una vida más digna y realizada según los proyectos de cada cual; comprendiendo que el acceso a ciertas informaciones socialmente relevantes es un mecanismo para evitar servilismos...

- la igualdad, incitando a conocer y disminuir la brecha digital, facilitando el acceso a las tecnologías comunicativas; delatando aquellos medios y plataformas que atentan contra la dignidad de personas y colectivos...

- la solidaridad, conociendo e implicando a la institución escolar en plataformas para la cooperación y el desarrollo; iniciando correspondencia electrónica con chicos y chicas de otros contextos; despertando formas de usar las redes sociales que conecten con las necesidades y derechos de los otros; colaborando vía electrónica con iniciativas para el desarrollo sostenible y el cuidado de la naturaleza...

- el diálogo y el respeto, reflexionando acerca de los beneficios y los límites de la tolerancia en sociedades democráticas; alentando la escucha activa, invitando a la apertura ante puntos de vista diferentes o no elegidos previamente por el alumnado en sus interacciones electrónicas; valorando en común las consecuencias de la violación de los derechos de autor en la red, o de las copias sin citar del texto de otra persona; reflexionando acerca del nuevo concepto de amistad que despiertan diferentes redes sociales, y las normas mínimas de cortesía y atención al otro que vale la pena usar en ellas...

\section{Ciudadanía digital}

El concepto de ciudadanía digital ha sido definido desde distintos enfoques. Una de las primeras definiciones tiene que ver con la idea que la ciudadanía digital puede definirse como las normas de comportamiento social que conciernen al uso de la tecnología (Ribble y Bailey, 2004). En este mismo sentido, Emejulu y McGregor (2016) conceptualizan la ciudadanía digital como una reacción a las tecnologías digitales que obligan a personas y grupos a adoptar habilidades y formas de ser para poder existir con éxito en un mundo de trabajo y ocio ininterrumpido y constante.

A nuestro modo de ver la conceptualización que hacen autores como Choi (2016), Gleason y Von Gillern (2018) y Kim y Choi (2018) es mucho más acertada -al no enfocarse como reacción sino como construcción- y está enfocada de manera 
más positiva, al afirmar que la ciudadanía digital incluye habilidades, pensamientos $\mathrm{y}$ acciones en las redes sociales que facilitan a las personas moverse en la red, comprenderse y participar tanto a nivel individual como grupal.

Por su parte, Robles (2009, p. 37) considera que los ciudadanos digitales son "aquellos ciudadanos que ejercitan todos o algunos de sus deberes y derechos a través de comunidades virtuales o de manera individual”. Esta definición sitúa el concepto de ciudadanía digital en el ámbito de lo valórico, del ejercicio de deberes y derechos, apuntando de este modo a la dimensión educativa (Lozano-Díaz y Fernández-Prados, 2019). Ello nos plantea de manera clara la necesidad de conocer en detalle los escenarios educativos, las modalidades que asume la construcción del saber, superando la mirada restrictiva sobre lo que las personas en formación deben hacer y saber para protegerse de los "peligros" de las redes sociales y las tecnologías digitales. En pocas palabras, la educación debe ser repensada en el marco de la sociedad digital (Owen, Grant, Sayers y Facer, 2006; Crockett y Churches, 2018), aprovechando las potencialidades de las tecnologías tanto en relación con el acceso al conocimiento como en el fomento de la participación cívica.

En este sentido, Casablancas, Schwartzmam y Burgui (2014) señalan dos componentes fundamentales. El primero tiene que ver con el hecho que educadores y educadoras retoman un papel fundamental como gestores de nuevos contenidos y también de prácticas creativas a la vez que formativas, en la participación e intervención en las redes, en escenarios digitales de intercambio y en el trabajo colectivo. El segundo componente está relacionado con la formación en el ámbito de la ciudadanía digital, lo que hace que los contenidos digitales se vinculen con nuevas visiones referidas al conocimiento, desde cuestiones de construcción colectiva, formatos múltiples y representaciones diversas, hasta las nociones de autoría junto con las perspectivas de la cultura libre como valor último, los modos de actuación responsable en los espacios digitales de participación social, la participación ciudadana y el activismo en la red.

A nuestro modo de ver, el concepto de ciudadanía digital y el desarrollo de la participación cívica a través de las redes sociales nos sitúa en el camino del aprendizaje-servicio digital, también denominado como e-aprendizaje-servicio. Veamos a continuación los motivos y algunas características.

\section{Tecnologías digitales y aprendizaje-servicio: ¿hacia el e-aprendizaje- servicio?}

Imaginemos un grupo de estudiantes de informática que diseñan una página web para una entidad sin ánimo de lucro, o un miembro de una asociación que envía a un grupo de estudiantes por vídeo-streaming información sobre las dinámicas de la entidad y las necesidades que tienen. Pensemos en un grupo de estudiantes que colaboran con un grupo de personas mayores para enseñarles los usos de los teléfonos móviles y las aplicaciones que tienen... 
Estos son ejemplos de situaciones reales que combinan el aprendizaje-servicio y las tecnologías digitales dando lugar a lo que algunos autores (Malvey, Hamby y Fottler, 2006; Dailey-Hebert, Donnelli-Sallee, y DiPadova-Stocks, 2008) han denominado e-aprendizaje-servicio (e-service-learning) y que ha sido definido como una pedagogía integrativa que compromete a los educandos mediante la tecnología en la indagación cívica, el servicio, la reflexión y la acción.

Hay diferentes motivos que han llevado a la aparición del e-aprendizaje-servicio (seguramente sería más correcto denominarlo aprendizaje-servicio mediado por tecnologías digitales, pero la primera es la denominación en inglés que se ha mantenido en las traducciones al español). El más importante de estos motivos es, sin duda, lo que se ha explicado en las líneas anteriores: la aparición de las tecnologías digitales y su uso intensivo y extensivo en los diferentes ámbitos de nuestra sociedad y, especialmente, en el ámbito educativo. Pero también merece ser señalado el papel que ha jugado la extensión de la educación a distancia (Bennett y Green 2001; Killian, 2004; Mironesco 2014); el modo en que las tecnologías digitales han sido usadas como herramientas para facilitar y mejorar las evidencias tanto de aprendizaje como de servicio (Burton, 2003; McWhorter, Delello y Roberts, 2016) y también la manera en que la tecnología puede promover la conciencia y el sentimiento de comunidad (Johnston, 1999; Swan, 2002).

En relación a la extensión de la educación a distancia, Bennett y Green (2001) señalan que la evolución de dicha modalidad educativa tiene la mejor alianza posible con el aprendizaje-servicio al reforzarse mutuamente, llegando a un número elevado de estudiantes y a mayores contextos de servicio, de manera que se establece una relación simbiótica. Killian (2004) remarca que en las asignaturas en modalidad virtual centradas en temas como la gestión, la evaluación de programas o los análisis políticos, el aprendizaje-servicio puede constituir la mejor pedagogía para conseguir los objetivos de aprendizaje de cada una de ellas. Por último, Mironesco (2014) señala que no existen muchas investigaciones sobre la realización de proyectos de aprendizaje-servicio por parte de estudiantes no tradicionales, como, por ejemplo, personas adultas con cargas familiares o estudiantes que trabajan y estudian a la vez. En estos casos, el e-aprendizaje-servicio puede facilitar el aprendizaje de los estudiantes, además de facilitar y promover prácticas de aprendizaje-servicio enraizadas en sus respectivas comunidades.

Todo ello permite afirmar que el aprendizaje-servicio mediado por tecnologías digitales es más que una curiosidad pedagógica o el empecinamiento tecnológico de algunos educadores, sino que representa el futuro del aprendizaje-servicio, tal y como afirman Waldner, McGorry y Widener (2012).

De hecho, seguramente es más adecuado referirnos ya a que determinadas prácticas de aprendizaje-servicio mediado por tecnologías digitales son una realidad. Ello es así porque las tecnologías digitales están presentes en diferentes momentos y actividades de los proyectos de aprendizaje-servicio. Así, por ejemplo, la comunicación entre las entidades comunitarias y educadores puede realizarse 
por correo electrónico, se pueden usar campus virtuales para sistematizar los aprendizajes del alumnado, o bien los foros electrónicos pueden ser usados para acompañar los procesos de práctica reflexiva.

Puig, Gijón, Martín y Rubio (2011) señalan que las condiciones pedagógicas principales que sustentan los proyectos de aprendizaje-servicio radican en que el aprendizaje debe basarse en la experiencia, desarrollarse de manera cooperativa y profundizarse mediante la práctica reflexiva. En este sentido, la tabla 1 muestra diferentes tecnologías agrupadas según el propósito con el que pueden ser usadas, aunque no siempre los límites son claros y algunas de las tecnologías y aplicaciones pueden ser usadas con más de una finalidad.

Tabla 1. Tecnologías digitales agrupadas según su propósito en un proyecto de aprendizajeservicio

\begin{tabular}{|l|l|}
\hline \multicolumn{1}{|c|}{ Propósito } & \multicolumn{1}{c|}{ Tecnologías } \\
\hline Comunicación & $\begin{array}{l}\text { Videoconferencias, chats, correo electrónico, foros virtuales, tablero de } \\
\text { anuncios de un campus virtual, Facebook, Twitter, listas de distribución }\end{array}$ \\
\hline $\begin{array}{l}\text { Búsqueda de } \\
\text { información }\end{array}$ & $\begin{array}{l}\text { Buscadores, buscadores temáticos, bases de datos en bibliotecas digitales, } \\
\text { Twitter, DropBox, RefWorks }\end{array}$ \\
\hline $\begin{array}{l}\text { Creación de } \\
\text { contenidos }\end{array}$ & Drive, Movie Maker, infografías, mapas conceptuales, nubes de palabras \\
\hline Colaboración & Wikis, blogs \\
\hline Reflexión & $\begin{array}{l}\text { Foros virtuales, wikis, blogs, infografías, mapas conceptuales, nubes de } \\
\text { palabras, grupos de Whastapp, narrativas digitales }\end{array}$ \\
\hline
\end{tabular}

Fuente: Elaboración propia

Estos diferentes usos de las tecnologías digitales nos permiten apuntar algo especialmente relevante: aquello más importante cuando nos planteamos introducir las tecnologías digitales en un proyecto de aprendizaje-servicio es que la tecnología siempretiene que ponerse al servicio del proyecto. Es bastante obvio, perola tecnología es solamente una herramienta que debe dar respuesta a los objetivos educativos y a la necesidad, y es importante no olvidarnos de este principio fundamental. Es por ello por lo que la selección de las tecnologías debe ser realizada de manera fundamentada en un doble criterio. En primer lugar, el criterio pedagógico, de manera que debemos plantearnos las respuestas a preguntas del tipo siguiente: ¿qué objetivo educativo tengo? ¿qué quiero hacer? ¿a qué necesidades debe dar respuesta la herramienta tecnológica? Y, en segundo lugar, debemos profundizar en el conocimiento de la tecnología escogida, analizando las bondades y limitaciones de cada una de las tecnologías y de sus posibles usos.

La tabla siguiente (adaptada de Strait y Nordyke, 2015) propone ejemplos de tecnologías digitales (tanto a nivel de hardware como de software) a usar en proyectos de aprendizaje-servicio. 
Tabla 2. Tecnologías digitales y ámbitos de aplicación en un proyecto de aprendizaje-servicio

\begin{tabular}{|l|l|l|}
\hline $\begin{array}{c}\text { Momentos del proyecto } \\
\text { de aprendizaje-servicio }\end{array}$ & \multicolumn{1}{|c|}{ Hardware } & \multicolumn{1}{|c|}{ Software (o función) } \\
\hline Actividad curricular & Ordenador, tableta & $\begin{array}{l}\text { Vídeos, libros electrónicos, } \\
\text { YouTube, Moodle, podcasts }\end{array}$ \\
\hline Servicio & $\begin{array}{l}\text { Teléfono móvil, ordenador, } \\
\text { tableta }\end{array}$ & $\begin{array}{l}\text { Páginas web, vídeos, } \\
\text { presentaciones }\end{array}$ \\
\hline Comunicación & $\begin{array}{l}\text { Teléfono móvil, ordenador, } \\
\text { tableta }\end{array}$ & $\begin{array}{l}\text { Correos electrónicos, } \\
\text { videoconferencias }\end{array}$ \\
\hline Seguimiento del proyecto & $\begin{array}{l}\text { Teléfono móvil, fotografía, } \\
\text { ordenador, tableta }\end{array}$ & $\begin{array}{l}\text { Cuestionarios on-line, foros } \\
\text { electrónicos }\end{array}$ \\
\hline $\begin{array}{l}\text { Reflexión de las personas } \\
\text { participantes }\end{array}$ & $\begin{array}{l}\text { Teléfono móvil, ordenador, } \\
\text { tableta }\end{array}$ & Blogs, vídeos, podcasts \\
\hline
\end{tabular}

Fuente: Adaptado de Strait y Nordyke, 2015

Hasta este momento hemos realizado una mirada en relación con el e-aprendizajeservicio en el que las tecnologías son una herramienta que media en los diferentes dinamismos fundamentales de los proyectos de aprendizaje-servicio. Pero es posible, según Waldner, McGorry y Widener (2010, 2012), plantear el e-aprendizaje-servicio como aquel aprendizaje-servicio que ocurre cuando o bien el dinamismo pedagógico o bien el dinamismo de servicio -o ambos- tienen lugar de manera virtual. En este sentido, los mismos autores afirman que el e-aprendizaje-servicio soluciona las limitaciones del aprendizaje-servicio tradicional y del aprendizaje virtual. Ello se debe al hecho que se libera al aprendizaje-servicio de las constricciones geográficas y también que da respuesta a una de las limitaciones fundamentales del aprendizaje virtual: la falta de interacción.

Los mismos autores proponen un modelo híbrido en el que o bien algunos aspectos del aprendizaje o bien algunos aspectos del servicio tienen lugar en la virtualidad, tal y como muestra la figura 1. 
Figura 1. Modelo híbrido de e-aprendizaje-servicio

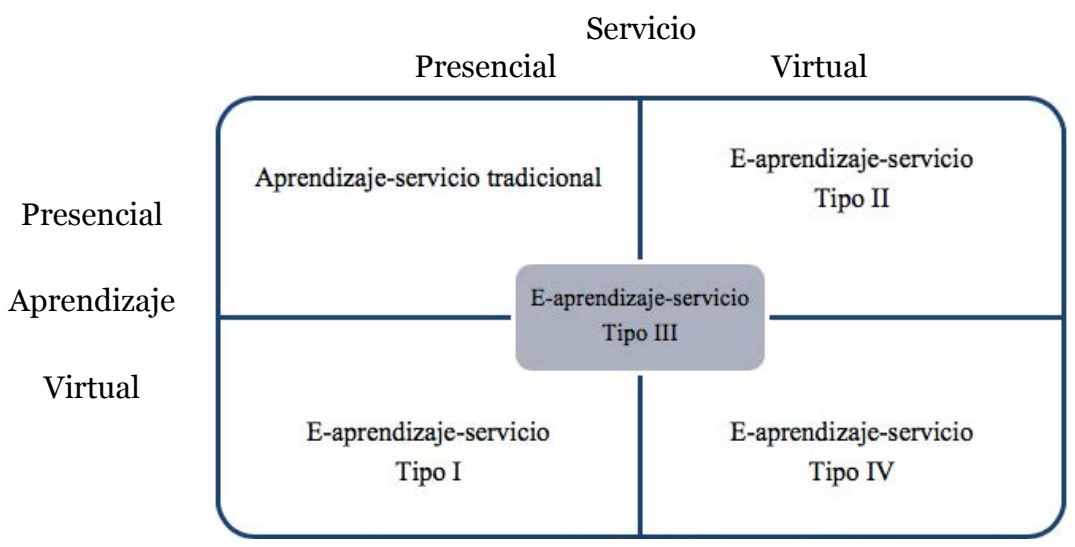

Fuente: Adaptado de Waldner, McGorry y Widener, (2012)

Tal y como puede verse, habría cuatro tipos de e-aprendizaje-servicio, desde el tipo I (servicio presencial y aprendizaje virtual) al tipo IV (completamente virtual, tanto el aprendizaje como el servicio), pasando por el tipo II (servicio virtual y aprendizaje presencial) y el tipo III (en el que tanto el aprendizaje como el servicio se realizan de manera semipresencial. La decisión sobre qué tipo de proyecto de aprendizaje-servicio podemos usar tiene que ver con los productos y resultados esperados. Por ejemplo, el Tipo II (aprendizaje presencial y servicio virtual) podría ser adecuado para proyectos en los que el servicio es el diseño de una página web como anteriormente se ha puesto como ejemplo. Pero, además, los tipos distintos de e-aprendizaje-servicio pueden tener diferentes resultados tanto en relación con el servicio como con el aprendizaje. Por ejemplo, en un proyecto de tipo IV (completamente virtual) es esperable una menor participación cívica, pero posiblemente, los resultados del proyecto pueden facilitar un desarrollo profesional más elevado, además que el alumnado puede aprender que existe la posibilidad de impactar positivamente en algunos aspectos de la realidad más allá del "simple" aprendizaje virtual. Finalmente, cada tipo de e-aprendizaje-servicio puede tener diferentes limitaciones y requerir diferentes técnicas para realización.

\section{Compromiso cívico, aprendizaje-servicio y tecnologías digitales: reflexiones finales}

A nuestro modo de ver, las tecnologías digitales y el aprendizaje-servicio van de la mano en la actualidad. Ello se debe a dos motivos; el primero tiene que ver -como se ha comentado ya- con el uso intensivo que hacen chicos y chicas de las tecnologías digitales en los diferentes ámbitos de su vida cotidiana. El segundo 
motivo está relacionado con las facilidades que ofrecen las tecnologías digitales y las redes sociales en relación con la participación. Así, de manera opuesta a la percepción extendida que la juventud de hoy en día está menos interesada en los temas sociales y políticos que en otras épocas y que tienen poco interés en ejercer su ciudadanía, los datos muestran que niños, niñas y jóvenes están comprometidos a través de las redes sociales en temáticas centradas en los intereses de la comunidad y en el desarrollo de su identidad ciudadana, de manera que su compromiso cívico puede aumentar en la medida que tengan nuevas y diferentes oportunidades para participar en la vida pública.

Unir los proyectos de aprendizaje-servicio y las tecnologías digitales facilita que jóvenes, niñas y niños experimenten de forma real aquello que es la solidaridad, la responsabilidad y el servicio a los otros, así como la satisfacción por los objetivos conseguidos. En definitiva, facilita conseguir el desarrollo de una ciudadanía responsable y consciente.

Las experiencias desarrolladas en este sentido son cada vez más numerosas, tanto en el ámbito formal en la enseñanza obligatoria (Jiménez, 2016; Sánchez y Solano, 2016) y en el nivel universitario (Cámara, Díaz y Ortega-Tudela, 2016; Duque, 2018; Fueyo y Belver, 2016; García-Gutiérrez, Ruíz-Corbella y Del Pozo, 2016; Mayor, 2019; Romero-Andonegi y Maíz, 2016; McWhorter, Delello y Roberts, 2016; Sánchez y López, 2016) como en el ámbito no formal (de Benito, Negre y Salinas, 2016).

Pero es que, además, las investigaciones muestran que el aprendizaje-servicio mediado por tecnologías digitales promueve el compromiso cívico y también mejora los procesos de aprendizaje (Rutti, La Bonte, Helms, Hervani y Sarkarat, 2016).

Por todo ello, queremos terminar con las recomendaciones ofrecidas por Strait y Sauer (2004) en un artículo que explica el nacimiento del e-aprendizaje-servicio: comenzar con un proyecto pequeño, capacitar a los estudiantes en el uso de las tecnologías digitales tanto en los momentos de aprendizaje como en los espacios de servicio, ponerse en contacto con entidades sociales y comunitarias, incluir la reflexión como una parte vital de las actividades $\mathrm{y}$, finalmente, prepararse para resultados inesperados.

\section{REFERENCIAS}

Álvaro, A., y Rubio, M. (2016). Las TIC en la participación política de los jóvenes. Madrid: Observatorio de la Juventud en España.

Banaji, S., y Buckingham, D. (2013). The civic web. Londres: MIT Press.

Bennett, G., y Green, F. P. (2001). Promoting service learning via online instruction. College Student Journal, 35(4), 491-497.
Buckingham, D. (2005). Educación en medios. Alfabetización, aprendizaje y cultura contemporánea. Madrid: Paidós.

Burton, E. (2003). Distance learning and service-learning in the accelerated format. New Directions for Adult and Continuing Education, 2003(97), 63-72.

Cámara, A., Díaz, E. M., y Ortega-Tudela, E. M. (2017). Aprendizaje-servicio en la universidad: ayudando a la escuela a 
atender a la diversidad a través de las TIC. Bordón, 69(3), 73-87.

Casablancas, S., Schwartzmam, G., y Burgui, S. (2014). Cambiando paradigmas sobre ciudadanía digital en el EDUCATÓN 2014, en Memorias del Congreso Iberoamericano de Ciencia, Tecnología, Innovación y Educación, Buenos Aires. Recuperado de http://www.oei.es/ historico/congres02014/memorias2014. php

Crockett, L. W., y Churches, A. (2018). Growing Global Digital Citizens. Better Practices That Build Better Learners. Bloomington: Solution Tree Press.

Choi, M. (2016). A Concept Analysis of Digital Citizenship for Democratic Citizenship Education in the Internet Age. Theory \& Research in Social Education, 44(4), 565-607. doi: 10.1080/00933104.2016.1210549.

Dailey-Hebert, A., Donnelli-Sallee, E., y DiPadova-Stocks, L. (2008). ServiceeLearning: Educating for citizenship. Charlotte: Information Age Publishing.

De Benito, B., Negre, F., y Salinas, M. (2016). Las TIC como estrategia para potenciar la motivación de un joven residente en un hospital. Estudio de caso. En R. Roig-Vila (Ed.), Tecnología, innovación $e$ investigación en los procesos de enseñanza-aprendizaje (2404-2412). Barcelona: Octaedro.

Duque, E. (2018). Evaluando una experiencia de aprendizaje-servicio en torno al aprendizaje de conceptos de la ciudadanía digital. RIDAS. Revista Iberoamericana de Aprendizaje Servicio, 5, 12-23.

Emejulu, A., y McGregor, C. (2016). Towards a radical digital citizenship in digital education. Critical Studies in Education, $60(1), 131-147$.

Fueyo, A., y Belver, J. L. (2016). Proyectos interdisciplinares de aprendizaje en servicio: la radio y el uso responsable de las TIC en la formación inicial de los profesionales de la educación. En R.
Roig-Vila (Ed.), Tecnología, innovación $e$ investigación en los procesos de enseñanza-aprendizaje (715-724). Barcelona: Octaedro.

Galindo, J. (2009). Ciudadanía digital. Signo y pensamiento, 28(54), 164-173.

García-Gutiérrez, J., Ruíz-Corbella, M., y Del Pozo, A. (2016). Cuando la comunidad se expande: ciudadanía global y aprendizajeservicio virtual. Educación y Diversidad, $10(2), 63-75$.

Gleason, B., y Von Gillern, S. (2018). Digital Citizenship with Social Media: Participatory Practices of Teaching and Learning in Secondary Education. Educational Technology \& Society, 21(1), 200-212.

Gozálvez, V. (2011). Educación para la ciudadanía democrática en la cultura digital. Comunicar, 36 (XVIII), 131-138.

Jiménez, M. Á. (2016). Realidad aumentada y aprendizaje-servicio para conocer $\mathrm{y}$ enriquecer nuestro entorno. En R. Roig-Vila (Ed.), Tecnología, innovación $e$ investigación en los procesos de enseñanza-aprendizaje (458-466). Barcelona: Octaedro.

Johnston, S. N. (1999). Practicing community through technology. En J. Deramo (Ed.), Best practices in cyber-serve: Integrating technology with servicelearning instruction (49-62). Blacksburg: Virginia Tech Service-learning Center.

Killian, J. (2004). Pedagogical experimentation: Combining traditional, distance, and service learning techniques. Journal of Public Affairs Education, 1O(3), 209-224.

Kim, M., y Choi, D. (2018). Development of Youth Digital Citizenship Scale and Implication for Educational Setting. Educational Technology \& Society, 21(1), 155-171.

Landow, G. P. (2009). Hipertexto 3.o: teoría crítica y nuevos medios en la era de la globalización. Barcelona: Paidós. 
Lozano-Díaz, A., y Fernández-Prados, J. S. (2019). Hacia una educación para la ciudadanía digital crítica y activa en la universidad. Revista Latinoamericana de Tecnología Educativa, 18(1), 175-187.

Malvey, D. M., Hamby, E. F., y Fottler, M. D. (2006). E-service learning: A pedagogic innovation for healthcare management education. Journal of Health Administration Education, 33(2), 181-198.

Martínez, M. (2011). De la brecha digital a la brecha cívica. Acceso a las tecnologías de la comunicación y participación ciudadana en la vida pública. Telos: Cuadernos de comunicación e innovación, 86, 24-36.

Mayor, D. (2019). El Aprendizaje-Servicio como práctica pedagógica para el desarrollo de competencias digitales y sociales del estudiantado universitario. Revista Iberoamericana de Educación, 8o(2), 9-28.

McCorkindale, T., DiStaso, M. W., y Sisco, H. F. (2013). How Millenials are engaging and building relationships with organisations on FaceBook. The Journal of Social Media in Society, 2(1), 66-87.

McWhorter, R., Delello, J., y Roberts, P. (2016). Giving Back: Exploring Service-Learning in an Online Learning Environment. Journal of Interactive Online Learning, 14(2), 80-99.

Mironesco, M. (2014). Using Service Learning to Enhance a Hybrid Course Curriculum in the "Politics of Food". MERLOT Journal of Online Learning and Teaching, 10 (3), 524-534.

Mossberger, K., Tolbert, C. J., y McNeal, R. S. (2008). Digital citizenship: The Internet, society, and participation. Cambridge: MIT Press.

Owen, M., Grant, L., Sayers, S., y Facer, K. (2006). Social software and learning. Bristol: Futurelab.

Puig, J. M., Gijón, M., Martín, X., y Rubio, L. (2011). Aprendizaje-servicio y Educación para la Ciudadanía. Revista de Educación (número extraordinario, dedicado a educación, valores y democracia), 45-67.

Reig, D. (2012). Disonancia cognitiva y apropiación delas TIC.TELOS: Cuadernos de comunicación e innovación, 90, 9-10.

Ribble, M., y Bailey, G. (2004). Digital Citizenship: Focus Questions for Implementation. Learning \& Leading with Technology, 32(2), 12-15.

Robles, J.M. (2009). Ciudadanía digital. Una introducción a un nuevo concepto de ciudadano. Barcelona: Editorial UOC.

Romero-Andonegi, A., y Maíz, I. (2016). Creando puentes: una experiencia para la formación en TIC del alumnado de Educación Infantil. En R. Roig-Vila (Ed.), Tecnología, innovación e investigación en los procesos de enseñanza-aprendizaje (1215-1221). Barcelona: Octaedro.

Rutti, R. M., LaBonte, J., Helms, M. M., Hervani, A. A., y Sarkarat, S. (2016). The service learning projects: Stakeholder benefits and potential class topics. Education + Training, 58(4), 422-438.

Sánchez, F., y López, D. (2016). El programa UPC-ReuTIlitza: reutilización de ordenadores como metodología de aprendizaje-servicio para incorporar sostenibilidad, cooperación y economía circular en estudios TIC. ReVisión, 9(3), 19-28.

Sánchez, M. M., y Solano, I. (2016). Repensando el uso de metodologías en educación infantil en situaciones enriquecidas con tecnologías. En R. Roig-Vila (Ed.), Tecnología, innovación $e$ investigación en los procesos de enseñanza-aprendizaje (3002-3015). Barcelona: Octaedro.

Strait, J., y Sauer, T. (2004). Constructing experiential learning for online courses: The birth of e-service. Educause Quarterly, 1, 62-65.

Strait, J., y Nordyke, K. J. (2015). E-ServiceLearning. Sterling: Stylus Publishing.

Swan, K. (2002) Building learning communities in online courses: The 
importance of interaction. Education, Communication and Information, 2(1), 23-49.

Tapscott, D. (1998). Creciendo en un entorno digital. Bogotá: Mc Graw-Hill.

Turkle, S. (1997). Life on the Screen: identity in the age of the Internet. New York: Simon \& Schuster.

Waldner, L. S., McGorry, S., y Widener, M. C. (2010). Extreme E-Service Learning
(XE-SL): E-Service Learning in the 100\% Online Course. MERLOT Journal of Online Learning and Teaching, 6(4), 839-851.

Waldner, L. S., McGorry, S., y Widener, M. C. (2012). E-Service-Learning: The Evolution of Service-Learning to Engage a Growing Online Student Population. Journal of Higher Education Outreach and Engagement, 16(2), 123-150.

\section{PERFIL ACADÉMICO Y PROFESIONAL DE LA AUTORA}

Anna Escofet. Profesora del Departamento de Teoría e Historia de la Educación de la Universidad de Barcelona. Miembro del grupo de investigación consolidado Entornos y Materiales de Aprendizaje. Sus áreas de interés son las tecnologías digitales, el aprendizaje-servicio, la brecha digital, la innovación y la educación superior.

E-mail: annaescofet@ub.edu

Dirección:

Facultad de Educación

Universidad de Barcelona

Vall d'Hebron, 171

Barcelona (España)

Fecha de recepción del artículo: 03/06/2019

Fecha de aceptación del artículo: 22/06/2019

Fecha de aprobación para maquetación: 11/08/2019 